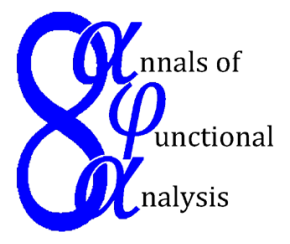

Ann. Funct. Anal. 7 (2016), no. 1, 61-75

http://dx.doi.org/10.1215/20088752-3320340

ISSN: 2008-8752 (electronic)

http://projecteuclid.org/afa

\title{
STRONG CONVERGENCE THEOREMS BY HYBRID METHODS FOR SEMIGROUPS OF NOT NECESSARILY CONTINUOUS MAPPINGS IN HILBERT SPACES
}

\author{
WATARU TAKAHASHI ${ }^{1 *}$ and MAKOTO TSUKADA ${ }^{2}$ \\ Dedicated to Professor Anthony To-Ming Lau \\ Communicated by B. Ricceri
}

\begin{abstract}
In this paper, we prove strong convergence theorems by two hybrid methods for semigroups of not necessarily continuous mappings in Hilbert spaces. Using these results, we prove strong convergence theorems for discrete semigroups generated by generalized hybrid mappings and semigroups of nonexpansive mappings in Hilbert spaces.
\end{abstract}

\section{INTRODUCTION}

Throughout this paper, we denote by $\mathbb{N}$ the set of positive integers and by $\mathbb{R}$ the set of real numbers. Let $H$ be a real Hilbert space, and let $C$ be a nonempty subset of $H$. Let $T$ be a mapping of $C$ into itself. We denote by $F(T)$ the set of fixed points of $T$ and by $A(T)$ the set of attractive points (see [15]) of $T$; that is,

(i) $F(T)=\{z \in C: T z=z\}$;

(ii) $A(T)=\{z \in H:\|T x-z\| \leq\|x-z\|, \forall x \in C\}$.

We know from [15] that $A(T)$ is always closed and convex. Kocourek, Takahashi, and Yao [7] defined a broad class of nonlinear mappings in a Hilbert space. A mapping $T: C \rightarrow C$ is called generalized hybrid (see [7]) if there exist $\alpha, \beta \in \mathbb{R}$ such

Copyright 2016 by the Tusi Mathematical Research Group.

Received Nov. 18, 2014; Accepted Mar. 25, 2015.

${ }^{*}$ Corresponding author.

2010 Mathematics Subject Classification. Primary 47H10; Secondary 47H20.

Keywords. generalized hybrid mapping, nonexpansive semigroup, invariant mean, fixed point, hybrid method. 


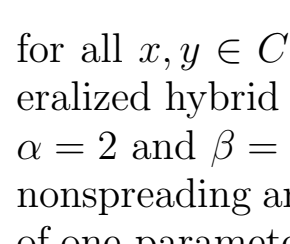

Ann. Funct. Anal. 7 (2016), no. 1, 61-75

http://dx.doi.org/10.1215/20088752-3320340

ISSN: 2008-8752 (electronic)

http://projecteuclid.org/afa

\title{
STRONG CONVERGENCE THEOREMS BY HYBRID METHODS FOR SEMIGROUPS OF NOT NECESSARILY CONTINUOUS MAPPINGS IN HILBERT SPACES
}

\author{
WATARU TAKAHASHI ${ }^{1 *}$ and MAKOTO TSUKADA ${ }^{2}$ \\ Dedicated to Professor Anthony To-Ming Lau \\ Communicated by B. Ricceri
}

\begin{abstract}
In this paper, we prove strong convergence theorems by two hybrid methods for semigroups of not necessarily continuous mappings in Hilbert spaces. Using these results, we prove strong convergence theorems for discrete semigroups generated by generalized hybrid mappings and semigroups of nonexpansive mappings in Hilbert spaces.
\end{abstract}

\section{INTRODUCTION}

Throughout this paper, we denote by $\mathbb{N}$ the set of positive integers and by $\mathbb{R}$ the set of real numbers. Let $H$ be a real Hilbert space, and let $C$ be a nonempty subset of $H$. Let $T$ be a mapping of $C$ into itself. We denote by $F(T)$ the set of fixed points of $T$ and by $A(T)$ the set of attractive points (see [15]) of $T$; that is,

(i) $F(T)=\{z \in C: T z=z\}$;

(ii) $A(T)=\{z \in H:\|T x-z\| \leq\|x-z\|, \forall x \in C\}$.

We know from [15] that $A(T)$ is always closed and convex. Kocourek, Takahashi, and Yao [7] defined a broad class of nonlinear mappings in a Hilbert space. A mapping $T: C \rightarrow C$ is called generalized hybrid (see [7]) if there exist $\alpha, \beta \in \mathbb{R}$ such

Copyright 2016 by the Tusi Mathematical Research Group.

Received Nov. 18, 2014; Accepted Mar. 25, 2015.

${ }^{*}$ Corresponding author.

2010 Mathematics Subject Classification. Primary 47H10; Secondary 47H20.

Keywords. generalized hybrid mapping, nonexpansive semigroup, invariant mean, fixed point, hybrid method. 


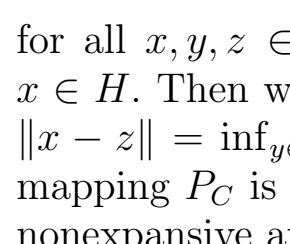

Ann. Funct. Anal. 7 (2016), no. 1, 61-75

http://dx.doi.org/10.1215/20088752-3320340

ISSN: 2008-8752 (electronic)

http://projecteuclid.org/afa

\title{
STRONG CONVERGENCE THEOREMS BY HYBRID METHODS FOR SEMIGROUPS OF NOT NECESSARILY CONTINUOUS MAPPINGS IN HILBERT SPACES
}

\author{
WATARU TAKAHASHI ${ }^{1 *}$ and MAKOTO TSUKADA ${ }^{2}$ \\ Dedicated to Professor Anthony To-Ming Lau \\ Communicated by B. Ricceri
}

\begin{abstract}
In this paper, we prove strong convergence theorems by two hybrid methods for semigroups of not necessarily continuous mappings in Hilbert spaces. Using these results, we prove strong convergence theorems for discrete semigroups generated by generalized hybrid mappings and semigroups of nonexpansive mappings in Hilbert spaces.
\end{abstract}

\section{INTRODUCTION}

Throughout this paper, we denote by $\mathbb{N}$ the set of positive integers and by $\mathbb{R}$ the set of real numbers. Let $H$ be a real Hilbert space, and let $C$ be a nonempty subset of $H$. Let $T$ be a mapping of $C$ into itself. We denote by $F(T)$ the set of fixed points of $T$ and by $A(T)$ the set of attractive points (see [15]) of $T$; that is,

(i) $F(T)=\{z \in C: T z=z\}$;

(ii) $A(T)=\{z \in H:\|T x-z\| \leq\|x-z\|, \forall x \in C\}$.

We know from [15] that $A(T)$ is always closed and convex. Kocourek, Takahashi, and Yao [7] defined a broad class of nonlinear mappings in a Hilbert space. A mapping $T: C \rightarrow C$ is called generalized hybrid (see [7]) if there exist $\alpha, \beta \in \mathbb{R}$ such

Copyright 2016 by the Tusi Mathematical Research Group.

Received Nov. 18, 2014; Accepted Mar. 25, 2015.

${ }^{*}$ Corresponding author.

2010 Mathematics Subject Classification. Primary 47H10; Secondary 47H20.

Keywords. generalized hybrid mapping, nonexpansive semigroup, invariant mean, fixed point, hybrid method. 


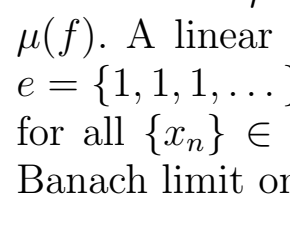

Ann. Funct. Anal. 7 (2016), no. 1, 61-75

http://dx.doi.org/10.1215/20088752-3320340

ISSN: 2008-8752 (electronic)

http://projecteuclid.org/afa

\title{
STRONG CONVERGENCE THEOREMS BY HYBRID METHODS FOR SEMIGROUPS OF NOT NECESSARILY CONTINUOUS MAPPINGS IN HILBERT SPACES
}

\author{
WATARU TAKAHASHI ${ }^{1 *}$ and MAKOTO TSUKADA ${ }^{2}$ \\ Dedicated to Professor Anthony To-Ming Lau \\ Communicated by B. Ricceri
}

\begin{abstract}
In this paper, we prove strong convergence theorems by two hybrid methods for semigroups of not necessarily continuous mappings in Hilbert spaces. Using these results, we prove strong convergence theorems for discrete semigroups generated by generalized hybrid mappings and semigroups of nonexpansive mappings in Hilbert spaces.
\end{abstract}

\section{INTRODUCTION}

Throughout this paper, we denote by $\mathbb{N}$ the set of positive integers and by $\mathbb{R}$ the set of real numbers. Let $H$ be a real Hilbert space, and let $C$ be a nonempty subset of $H$. Let $T$ be a mapping of $C$ into itself. We denote by $F(T)$ the set of fixed points of $T$ and by $A(T)$ the set of attractive points (see [15]) of $T$; that is,

(i) $F(T)=\{z \in C: T z=z\}$;

(ii) $A(T)=\{z \in H:\|T x-z\| \leq\|x-z\|, \forall x \in C\}$.

We know from [15] that $A(T)$ is always closed and convex. Kocourek, Takahashi, and Yao [7] defined a broad class of nonlinear mappings in a Hilbert space. A mapping $T: C \rightarrow C$ is called generalized hybrid (see [7]) if there exist $\alpha, \beta \in \mathbb{R}$ such

Copyright 2016 by the Tusi Mathematical Research Group.

Received Nov. 18, 2014; Accepted Mar. 25, 2015.

${ }^{*}$ Corresponding author.

2010 Mathematics Subject Classification. Primary 47H10; Secondary 47H20.

Keywords. generalized hybrid mapping, nonexpansive semigroup, invariant mean, fixed point, hybrid method. 


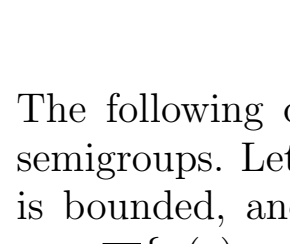

Ann. Funct. Anal. 7 (2016), no. 1, 61-75

http://dx.doi.org/10.1215/20088752-3320340

ISSN: 2008-8752 (electronic)

http://projecteuclid.org/afa

\title{
STRONG CONVERGENCE THEOREMS BY HYBRID METHODS FOR SEMIGROUPS OF NOT NECESSARILY CONTINUOUS MAPPINGS IN HILBERT SPACES
}

\author{
WATARU TAKAHASHI ${ }^{1 *}$ and MAKOTO TSUKADA ${ }^{2}$ \\ Dedicated to Professor Anthony To-Ming Lau \\ Communicated by B. Ricceri
}

\begin{abstract}
In this paper, we prove strong convergence theorems by two hybrid methods for semigroups of not necessarily continuous mappings in Hilbert spaces. Using these results, we prove strong convergence theorems for discrete semigroups generated by generalized hybrid mappings and semigroups of nonexpansive mappings in Hilbert spaces.
\end{abstract}

\section{INTRODUCTION}

Throughout this paper, we denote by $\mathbb{N}$ the set of positive integers and by $\mathbb{R}$ the set of real numbers. Let $H$ be a real Hilbert space, and let $C$ be a nonempty subset of $H$. Let $T$ be a mapping of $C$ into itself. We denote by $F(T)$ the set of fixed points of $T$ and by $A(T)$ the set of attractive points (see [15]) of $T$; that is,

(i) $F(T)=\{z \in C: T z=z\}$;

(ii) $A(T)=\{z \in H:\|T x-z\| \leq\|x-z\|, \forall x \in C\}$.

We know from [15] that $A(T)$ is always closed and convex. Kocourek, Takahashi, and Yao [7] defined a broad class of nonlinear mappings in a Hilbert space. A mapping $T: C \rightarrow C$ is called generalized hybrid (see [7]) if there exist $\alpha, \beta \in \mathbb{R}$ such

Copyright 2016 by the Tusi Mathematical Research Group.

Received Nov. 18, 2014; Accepted Mar. 25, 2015.

${ }^{*}$ Corresponding author.

2010 Mathematics Subject Classification. Primary 47H10; Secondary 47H20.

Keywords. generalized hybrid mapping, nonexpansive semigroup, invariant mean, fixed point, hybrid method. 


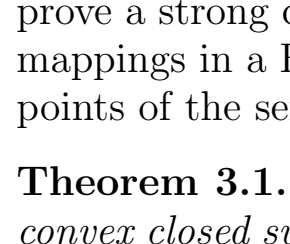

Ann. Funct. Anal. 7 (2016), no. 1, 61-75

http://dx.doi.org/10.1215/20088752-3320340

ISSN: 2008-8752 (electronic)

http://projecteuclid.org/afa

\title{
STRONG CONVERGENCE THEOREMS BY HYBRID METHODS FOR SEMIGROUPS OF NOT NECESSARILY CONTINUOUS MAPPINGS IN HILBERT SPACES
}

\author{
WATARU TAKAHASHI ${ }^{1 *}$ and MAKOTO TSUKADA ${ }^{2}$ \\ Dedicated to Professor Anthony To-Ming Lau \\ Communicated by B. Ricceri
}

\begin{abstract}
In this paper, we prove strong convergence theorems by two hybrid methods for semigroups of not necessarily continuous mappings in Hilbert spaces. Using these results, we prove strong convergence theorems for discrete semigroups generated by generalized hybrid mappings and semigroups of nonexpansive mappings in Hilbert spaces.
\end{abstract}

\section{INTRODUCTION}

Throughout this paper, we denote by $\mathbb{N}$ the set of positive integers and by $\mathbb{R}$ the set of real numbers. Let $H$ be a real Hilbert space, and let $C$ be a nonempty subset of $H$. Let $T$ be a mapping of $C$ into itself. We denote by $F(T)$ the set of fixed points of $T$ and by $A(T)$ the set of attractive points (see [15]) of $T$; that is,

(i) $F(T)=\{z \in C: T z=z\}$;

(ii) $A(T)=\{z \in H:\|T x-z\| \leq\|x-z\|, \forall x \in C\}$.

We know from [15] that $A(T)$ is always closed and convex. Kocourek, Takahashi, and Yao [7] defined a broad class of nonlinear mappings in a Hilbert space. A mapping $T: C \rightarrow C$ is called generalized hybrid (see [7]) if there exist $\alpha, \beta \in \mathbb{R}$ such

Copyright 2016 by the Tusi Mathematical Research Group.

Received Nov. 18, 2014; Accepted Mar. 25, 2015.

${ }^{*}$ Corresponding author.

2010 Mathematics Subject Classification. Primary 47H10; Secondary 47H20.

Keywords. generalized hybrid mapping, nonexpansive semigroup, invariant mean, fixed point, hybrid method. 


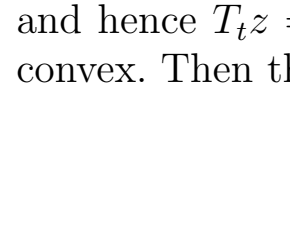

Ann. Funct. Anal. 7 (2016), no. 1, 61-75

http://dx.doi.org/10.1215/20088752-3320340

ISSN: 2008-8752 (electronic)

http://projecteuclid.org/afa

\title{
STRONG CONVERGENCE THEOREMS BY HYBRID METHODS FOR SEMIGROUPS OF NOT NECESSARILY CONTINUOUS MAPPINGS IN HILBERT SPACES
}

\author{
WATARU TAKAHASHI ${ }^{1 *}$ and MAKOTO TSUKADA ${ }^{2}$ \\ Dedicated to Professor Anthony To-Ming Lau \\ Communicated by B. Ricceri
}

\begin{abstract}
In this paper, we prove strong convergence theorems by two hybrid methods for semigroups of not necessarily continuous mappings in Hilbert spaces. Using these results, we prove strong convergence theorems for discrete semigroups generated by generalized hybrid mappings and semigroups of nonexpansive mappings in Hilbert spaces.
\end{abstract}

\section{INTRODUCTION}

Throughout this paper, we denote by $\mathbb{N}$ the set of positive integers and by $\mathbb{R}$ the set of real numbers. Let $H$ be a real Hilbert space, and let $C$ be a nonempty subset of $H$. Let $T$ be a mapping of $C$ into itself. We denote by $F(T)$ the set of fixed points of $T$ and by $A(T)$ the set of attractive points (see [15]) of $T$; that is,

(i) $F(T)=\{z \in C: T z=z\}$;

(ii) $A(T)=\{z \in H:\|T x-z\| \leq\|x-z\|, \forall x \in C\}$.

We know from [15] that $A(T)$ is always closed and convex. Kocourek, Takahashi, and Yao [7] defined a broad class of nonlinear mappings in a Hilbert space. A mapping $T: C \rightarrow C$ is called generalized hybrid (see [7]) if there exist $\alpha, \beta \in \mathbb{R}$ such

Copyright 2016 by the Tusi Mathematical Research Group.

Received Nov. 18, 2014; Accepted Mar. 25, 2015.

${ }^{*}$ Corresponding author.

2010 Mathematics Subject Classification. Primary 47H10; Secondary 47H20.

Keywords. generalized hybrid mapping, nonexpansive semigroup, invariant mean, fixed point, hybrid method. 


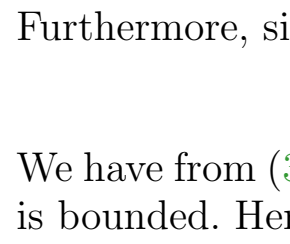

Ann. Funct. Anal. 7 (2016), no. 1, 61-75

http://dx.doi.org/10.1215/20088752-3320340

ISSN: 2008-8752 (electronic)

http://projecteuclid.org/afa

\title{
STRONG CONVERGENCE THEOREMS BY HYBRID METHODS FOR SEMIGROUPS OF NOT NECESSARILY CONTINUOUS MAPPINGS IN HILBERT SPACES
}

\author{
WATARU TAKAHASHI ${ }^{1 *}$ and MAKOTO TSUKADA ${ }^{2}$ \\ Dedicated to Professor Anthony To-Ming Lau \\ Communicated by B. Ricceri
}

\begin{abstract}
In this paper, we prove strong convergence theorems by two hybrid methods for semigroups of not necessarily continuous mappings in Hilbert spaces. Using these results, we prove strong convergence theorems for discrete semigroups generated by generalized hybrid mappings and semigroups of nonexpansive mappings in Hilbert spaces.
\end{abstract}

\section{INTRODUCTION}

Throughout this paper, we denote by $\mathbb{N}$ the set of positive integers and by $\mathbb{R}$ the set of real numbers. Let $H$ be a real Hilbert space, and let $C$ be a nonempty subset of $H$. Let $T$ be a mapping of $C$ into itself. We denote by $F(T)$ the set of fixed points of $T$ and by $A(T)$ the set of attractive points (see [15]) of $T$; that is,

(i) $F(T)=\{z \in C: T z=z\}$;

(ii) $A(T)=\{z \in H:\|T x-z\| \leq\|x-z\|, \forall x \in C\}$.

We know from [15] that $A(T)$ is always closed and convex. Kocourek, Takahashi, and Yao [7] defined a broad class of nonlinear mappings in a Hilbert space. A mapping $T: C \rightarrow C$ is called generalized hybrid (see [7]) if there exist $\alpha, \beta \in \mathbb{R}$ such

Copyright 2016 by the Tusi Mathematical Research Group.

Received Nov. 18, 2014; Accepted Mar. 25, 2015.

${ }^{*}$ Corresponding author.

2010 Mathematics Subject Classification. Primary 47H10; Secondary 47H20.

Keywords. generalized hybrid mapping, nonexpansive semigroup, invariant mean, fixed point, hybrid method. 


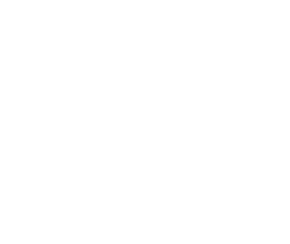

Ann. Funct. Anal. 7 (2016), no. 1, 61-75

http://dx.doi.org/10.1215/20088752-3320340

ISSN: 2008-8752 (electronic)

http://projecteuclid.org/afa

\title{
STRONG CONVERGENCE THEOREMS BY HYBRID METHODS FOR SEMIGROUPS OF NOT NECESSARILY CONTINUOUS MAPPINGS IN HILBERT SPACES
}

\author{
WATARU TAKAHASHI ${ }^{1 *}$ and MAKOTO TSUKADA ${ }^{2}$ \\ Dedicated to Professor Anthony To-Ming Lau \\ Communicated by B. Ricceri
}

\begin{abstract}
In this paper, we prove strong convergence theorems by two hybrid methods for semigroups of not necessarily continuous mappings in Hilbert spaces. Using these results, we prove strong convergence theorems for discrete semigroups generated by generalized hybrid mappings and semigroups of nonexpansive mappings in Hilbert spaces.
\end{abstract}

\section{INTRODUCTION}

Throughout this paper, we denote by $\mathbb{N}$ the set of positive integers and by $\mathbb{R}$ the set of real numbers. Let $H$ be a real Hilbert space, and let $C$ be a nonempty subset of $H$. Let $T$ be a mapping of $C$ into itself. We denote by $F(T)$ the set of fixed points of $T$ and by $A(T)$ the set of attractive points (see [15]) of $T$; that is,

(i) $F(T)=\{z \in C: T z=z\}$;

(ii) $A(T)=\{z \in H:\|T x-z\| \leq\|x-z\|, \forall x \in C\}$.

We know from [15] that $A(T)$ is always closed and convex. Kocourek, Takahashi, and Yao [7] defined a broad class of nonlinear mappings in a Hilbert space. A mapping $T: C \rightarrow C$ is called generalized hybrid (see [7]) if there exist $\alpha, \beta \in \mathbb{R}$ such

Copyright 2016 by the Tusi Mathematical Research Group.

Received Nov. 18, 2014; Accepted Mar. 25, 2015.

${ }^{*}$ Corresponding author.

2010 Mathematics Subject Classification. Primary 47H10; Secondary 47H20.

Keywords. generalized hybrid mapping, nonexpansive semigroup, invariant mean, fixed point, hybrid method. 


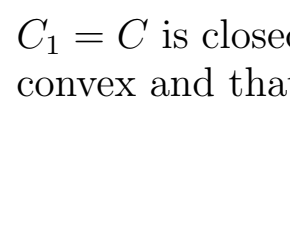

Ann. Funct. Anal. 7 (2016), no. 1, 61-75

http://dx.doi.org/10.1215/20088752-3320340

ISSN: 2008-8752 (electronic)

http://projecteuclid.org/afa

\title{
STRONG CONVERGENCE THEOREMS BY HYBRID METHODS FOR SEMIGROUPS OF NOT NECESSARILY CONTINUOUS MAPPINGS IN HILBERT SPACES
}

\author{
WATARU TAKAHASHI ${ }^{1 *}$ and MAKOTO TSUKADA ${ }^{2}$ \\ Dedicated to Professor Anthony To-Ming Lau \\ Communicated by B. Ricceri
}

\begin{abstract}
In this paper, we prove strong convergence theorems by two hybrid methods for semigroups of not necessarily continuous mappings in Hilbert spaces. Using these results, we prove strong convergence theorems for discrete semigroups generated by generalized hybrid mappings and semigroups of nonexpansive mappings in Hilbert spaces.
\end{abstract}

\section{INTRODUCTION}

Throughout this paper, we denote by $\mathbb{N}$ the set of positive integers and by $\mathbb{R}$ the set of real numbers. Let $H$ be a real Hilbert space, and let $C$ be a nonempty subset of $H$. Let $T$ be a mapping of $C$ into itself. We denote by $F(T)$ the set of fixed points of $T$ and by $A(T)$ the set of attractive points (see [15]) of $T$; that is,

(i) $F(T)=\{z \in C: T z=z\}$;

(ii) $A(T)=\{z \in H:\|T x-z\| \leq\|x-z\|, \forall x \in C\}$.

We know from [15] that $A(T)$ is always closed and convex. Kocourek, Takahashi, and Yao [7] defined a broad class of nonlinear mappings in a Hilbert space. A mapping $T: C \rightarrow C$ is called generalized hybrid (see [7]) if there exist $\alpha, \beta \in \mathbb{R}$ such

Copyright 2016 by the Tusi Mathematical Research Group.

Received Nov. 18, 2014; Accepted Mar. 25, 2015.

${ }^{*}$ Corresponding author.

2010 Mathematics Subject Classification. Primary 47H10; Secondary 47H20.

Keywords. generalized hybrid mapping, nonexpansive semigroup, invariant mean, fixed point, hybrid method. 


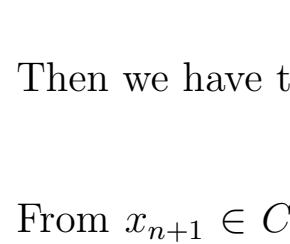

Ann. Funct. Anal. 7 (2016), no. 1, 61-75

http://dx.doi.org/10.1215/20088752-3320340

ISSN: 2008-8752 (electronic)

http://projecteuclid.org/afa

\title{
STRONG CONVERGENCE THEOREMS BY HYBRID METHODS FOR SEMIGROUPS OF NOT NECESSARILY CONTINUOUS MAPPINGS IN HILBERT SPACES
}

\author{
WATARU TAKAHASHI ${ }^{1 *}$ and MAKOTO TSUKADA ${ }^{2}$ \\ Dedicated to Professor Anthony To-Ming Lau \\ Communicated by B. Ricceri
}

\begin{abstract}
In this paper, we prove strong convergence theorems by two hybrid methods for semigroups of not necessarily continuous mappings in Hilbert spaces. Using these results, we prove strong convergence theorems for discrete semigroups generated by generalized hybrid mappings and semigroups of nonexpansive mappings in Hilbert spaces.
\end{abstract}

\section{INTRODUCTION}

Throughout this paper, we denote by $\mathbb{N}$ the set of positive integers and by $\mathbb{R}$ the set of real numbers. Let $H$ be a real Hilbert space, and let $C$ be a nonempty subset of $H$. Let $T$ be a mapping of $C$ into itself. We denote by $F(T)$ the set of fixed points of $T$ and by $A(T)$ the set of attractive points (see [15]) of $T$; that is,

(i) $F(T)=\{z \in C: T z=z\}$;

(ii) $A(T)=\{z \in H:\|T x-z\| \leq\|x-z\|, \forall x \in C\}$.

We know from [15] that $A(T)$ is always closed and convex. Kocourek, Takahashi, and Yao [7] defined a broad class of nonlinear mappings in a Hilbert space. A mapping $T: C \rightarrow C$ is called generalized hybrid (see [7]) if there exist $\alpha, \beta \in \mathbb{R}$ such

Copyright 2016 by the Tusi Mathematical Research Group.

Received Nov. 18, 2014; Accepted Mar. 25, 2015.

${ }^{*}$ Corresponding author.

2010 Mathematics Subject Classification. Primary 47H10; Secondary 47H20.

Keywords. generalized hybrid mapping, nonexpansive semigroup, invariant mean, fixed point, hybrid method. 


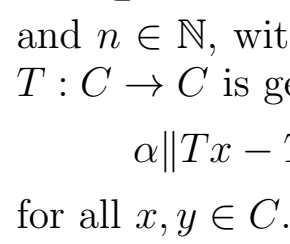

Ann. Funct. Anal. 7 (2016), no. 1, 61-75

http://dx.doi.org/10.1215/20088752-3320340

ISSN: 2008-8752 (electronic)

http://projecteuclid.org/afa

\title{
STRONG CONVERGENCE THEOREMS BY HYBRID METHODS FOR SEMIGROUPS OF NOT NECESSARILY CONTINUOUS MAPPINGS IN HILBERT SPACES
}

\author{
WATARU TAKAHASHI ${ }^{1 *}$ and MAKOTO TSUKADA ${ }^{2}$ \\ Dedicated to Professor Anthony To-Ming Lau \\ Communicated by B. Ricceri
}

\begin{abstract}
In this paper, we prove strong convergence theorems by two hybrid methods for semigroups of not necessarily continuous mappings in Hilbert spaces. Using these results, we prove strong convergence theorems for discrete semigroups generated by generalized hybrid mappings and semigroups of nonexpansive mappings in Hilbert spaces.
\end{abstract}

\section{INTRODUCTION}

Throughout this paper, we denote by $\mathbb{N}$ the set of positive integers and by $\mathbb{R}$ the set of real numbers. Let $H$ be a real Hilbert space, and let $C$ be a nonempty subset of $H$. Let $T$ be a mapping of $C$ into itself. We denote by $F(T)$ the set of fixed points of $T$ and by $A(T)$ the set of attractive points (see [15]) of $T$; that is,

(i) $F(T)=\{z \in C: T z=z\}$;

(ii) $A(T)=\{z \in H:\|T x-z\| \leq\|x-z\|, \forall x \in C\}$.

We know from [15] that $A(T)$ is always closed and convex. Kocourek, Takahashi, and Yao [7] defined a broad class of nonlinear mappings in a Hilbert space. A mapping $T: C \rightarrow C$ is called generalized hybrid (see [7]) if there exist $\alpha, \beta \in \mathbb{R}$ such

Copyright 2016 by the Tusi Mathematical Research Group.

Received Nov. 18, 2014; Accepted Mar. 25, 2015.

${ }^{*}$ Corresponding author.

2010 Mathematics Subject Classification. Primary 47H10; Secondary 47H20.

Keywords. generalized hybrid mapping, nonexpansive semigroup, invariant mean, fixed point, hybrid method. 


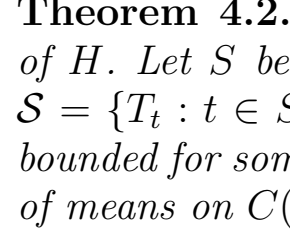

Ann. Funct. Anal. 7 (2016), no. 1, 61-75

http://dx.doi.org/10.1215/20088752-3320340

ISSN: 2008-8752 (electronic)

http://projecteuclid.org/afa

\title{
STRONG CONVERGENCE THEOREMS BY HYBRID METHODS FOR SEMIGROUPS OF NOT NECESSARILY CONTINUOUS MAPPINGS IN HILBERT SPACES
}

\author{
WATARU TAKAHASHI ${ }^{1 *}$ and MAKOTO TSUKADA ${ }^{2}$ \\ Dedicated to Professor Anthony To-Ming Lau \\ Communicated by B. Ricceri
}

\begin{abstract}
In this paper, we prove strong convergence theorems by two hybrid methods for semigroups of not necessarily continuous mappings in Hilbert spaces. Using these results, we prove strong convergence theorems for discrete semigroups generated by generalized hybrid mappings and semigroups of nonexpansive mappings in Hilbert spaces.
\end{abstract}

\section{INTRODUCTION}

Throughout this paper, we denote by $\mathbb{N}$ the set of positive integers and by $\mathbb{R}$ the set of real numbers. Let $H$ be a real Hilbert space, and let $C$ be a nonempty subset of $H$. Let $T$ be a mapping of $C$ into itself. We denote by $F(T)$ the set of fixed points of $T$ and by $A(T)$ the set of attractive points (see [15]) of $T$; that is,

(i) $F(T)=\{z \in C: T z=z\}$;

(ii) $A(T)=\{z \in H:\|T x-z\| \leq\|x-z\|, \forall x \in C\}$.

We know from [15] that $A(T)$ is always closed and convex. Kocourek, Takahashi, and Yao [7] defined a broad class of nonlinear mappings in a Hilbert space. A mapping $T: C \rightarrow C$ is called generalized hybrid (see [7]) if there exist $\alpha, \beta \in \mathbb{R}$ such

Copyright 2016 by the Tusi Mathematical Research Group.

Received Nov. 18, 2014; Accepted Mar. 25, 2015.

${ }^{*}$ Corresponding author.

2010 Mathematics Subject Classification. Primary 47H10; Secondary 47H20.

Keywords. generalized hybrid mapping, nonexpansive semigroup, invariant mean, fixed point, hybrid method. 


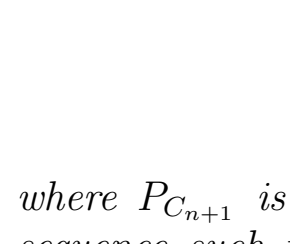

Ann. Funct. Anal. 7 (2016), no. 1, 61-75

http://dx.doi.org/10.1215/20088752-3320340

ISSN: 2008-8752 (electronic)

http://projecteuclid.org/afa

\title{
STRONG CONVERGENCE THEOREMS BY HYBRID METHODS FOR SEMIGROUPS OF NOT NECESSARILY CONTINUOUS MAPPINGS IN HILBERT SPACES
}

\author{
WATARU TAKAHASHI ${ }^{1 *}$ and MAKOTO TSUKADA ${ }^{2}$ \\ Dedicated to Professor Anthony To-Ming Lau \\ Communicated by B. Ricceri
}

\begin{abstract}
In this paper, we prove strong convergence theorems by two hybrid methods for semigroups of not necessarily continuous mappings in Hilbert spaces. Using these results, we prove strong convergence theorems for discrete semigroups generated by generalized hybrid mappings and semigroups of nonexpansive mappings in Hilbert spaces.
\end{abstract}

\section{INTRODUCTION}

Throughout this paper, we denote by $\mathbb{N}$ the set of positive integers and by $\mathbb{R}$ the set of real numbers. Let $H$ be a real Hilbert space, and let $C$ be a nonempty subset of $H$. Let $T$ be a mapping of $C$ into itself. We denote by $F(T)$ the set of fixed points of $T$ and by $A(T)$ the set of attractive points (see [15]) of $T$; that is,

(i) $F(T)=\{z \in C: T z=z\}$;

(ii) $A(T)=\{z \in H:\|T x-z\| \leq\|x-z\|, \forall x \in C\}$.

We know from [15] that $A(T)$ is always closed and convex. Kocourek, Takahashi, and Yao [7] defined a broad class of nonlinear mappings in a Hilbert space. A mapping $T: C \rightarrow C$ is called generalized hybrid (see [7]) if there exist $\alpha, \beta \in \mathbb{R}$ such

Copyright 2016 by the Tusi Mathematical Research Group.

Received Nov. 18, 2014; Accepted Mar. 25, 2015.

${ }^{*}$ Corresponding author.

2010 Mathematics Subject Classification. Primary 47H10; Secondary 47H20.

Keywords. generalized hybrid mapping, nonexpansive semigroup, invariant mean, fixed point, hybrid method. 


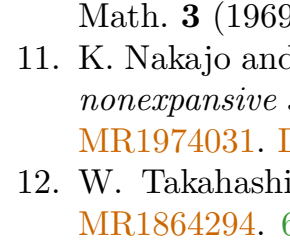

Ann. Funct. Anal. 7 (2016), no. 1, 61-75

http://dx.doi.org/10.1215/20088752-3320340

ISSN: 2008-8752 (electronic)

http://projecteuclid.org/afa

\title{
STRONG CONVERGENCE THEOREMS BY HYBRID METHODS FOR SEMIGROUPS OF NOT NECESSARILY CONTINUOUS MAPPINGS IN HILBERT SPACES
}

\author{
WATARU TAKAHASHI ${ }^{1 *}$ and MAKOTO TSUKADA ${ }^{2}$ \\ Dedicated to Professor Anthony To-Ming Lau \\ Communicated by B. Ricceri
}

\begin{abstract}
In this paper, we prove strong convergence theorems by two hybrid methods for semigroups of not necessarily continuous mappings in Hilbert spaces. Using these results, we prove strong convergence theorems for discrete semigroups generated by generalized hybrid mappings and semigroups of nonexpansive mappings in Hilbert spaces.
\end{abstract}

\section{INTRODUCTION}

Throughout this paper, we denote by $\mathbb{N}$ the set of positive integers and by $\mathbb{R}$ the set of real numbers. Let $H$ be a real Hilbert space, and let $C$ be a nonempty subset of $H$. Let $T$ be a mapping of $C$ into itself. We denote by $F(T)$ the set of fixed points of $T$ and by $A(T)$ the set of attractive points (see [15]) of $T$; that is,

(i) $F(T)=\{z \in C: T z=z\}$;

(ii) $A(T)=\{z \in H:\|T x-z\| \leq\|x-z\|, \forall x \in C\}$.

We know from [15] that $A(T)$ is always closed and convex. Kocourek, Takahashi, and Yao [7] defined a broad class of nonlinear mappings in a Hilbert space. A mapping $T: C \rightarrow C$ is called generalized hybrid (see [7]) if there exist $\alpha, \beta \in \mathbb{R}$ such

Copyright 2016 by the Tusi Mathematical Research Group.

Received Nov. 18, 2014; Accepted Mar. 25, 2015.

${ }^{*}$ Corresponding author.

2010 Mathematics Subject Classification. Primary 47H10; Secondary 47H20.

Keywords. generalized hybrid mapping, nonexpansive semigroup, invariant mean, fixed point, hybrid method. 